\title{
Desempenho agronômico de cultivares de amendoim em diferentes épocas de semeadura nas condições de Sorriso- MT
}

Submetido - 27 jul. $2020 \quad$ Aprovado - 10 set. $2020 \quad$ Publicado - 14 out. 2020
dol http://dx.doi.org/10.17648/sas.v1i2.41

Dácio Olibone $\mathbb{D}$

Professor do Instituto Federal de Educação, Ciência e Tecnologia de Mato Grosso - IFMT, Sorriso, MT, e-mail: dacio.olibone@srs.ifmt.edu.br.

Fabio Pedro Werner (iD

Discente de Engenharia Agronômica, Instituto Federal de Educação, Ciência e Tecnologia de Mato Grosso - IFMT, Sorriso, MT, e-mail: fabiowerner1@gmail.com.

Programa de Melhoramento do Amendoim - Embrapa, Santo Antônio de Goiás, GO, e-mail: jair.heuert@embrapa.br.

Laerte Gustavo Pivetta iD Professor do Instituto Federal de Educação, Ciência e Tecnologia de Mato Grosso - IFMT, Sorriso, MT, e-mail: laerte.pivetta@srs.ifmt.edu.br.

Discente de Engenharia Agronômica, Instituto Federal de Educação, Ciência e Tecnologia de Mato Grosso - IFMT, Sorriso, MT, e-mail: marianithamariano@gmail.com.

Discente de Agronomia do IFMT Campus São Vicente - Centro de Referência de Campo Verde, Campo Verde, MT, e-mail: maxuelfellipe90@gmail.com.

\section{RESUMO}

Produtores do Mato Grosso tem demonstrado interesse no cultivo do amendoim, porém as informações geradas pela pesquisa ainda são insuficientes sobre épocas e cultivares. 0 objetivo deste estudo é determinar a melhor época de semeadura para 5 cultivares de amendoim nas condições de Sorriso-MT. O experimento foi conduzido em área experimental do Instituto Federal de Educação, Ciência e Tecnologia - IFMT Campus Sorriso, localizado no município de Sorriso-MT. O delineamento experimental utilizado foi em blocos casualizados, cada tratamento com 3 repetições, em esquema fatorial 5 x 5 , sendo 5 épocas de semeadura $(07 / 11 / 2019,28 / 11 / 2019,19 / 12 / 2019,09 / 01 / 2020$ e 30/01/2020) e 5 cultivares de amendoim (BRS 421 OL, BRS 423 OL, BRS 425 OL, desenvolvidas pelo Programa de Melhoramento do Amendoim da Embrapa e as cultivares EC 98 AO e Granoleico desenvolvidas pelo Criadero EI Carmen da Argentina. As parcelas foram compostas por 4 linhas de 4 metros de comprimento e espaçamento de 0,90 m. Aos 135 dias após a emergência foram colhidas as plantas da área útil para determinação da produtividade de amendoim em vagens e a massa de 100 grãos. Nas condições experimentais em que foi desenvolvida esta pesquisa, pode-se concluir que a cultivar BRS 425 OL e Granoleico obtiveram as maiores produtividades na época de 07 de novembro, enquanto a EC 98 AO foi mais produtiva em 30 de janeiro em comparação às demais épocas de semeadura. As cultivares BRS 421 OL e BRS 423 OL não obtiveram diferença significativa quanto a produtividade nas diferentes épocas de semeadura, apresentando resultados similares em todas as épocas de semeadura, demonstrando possível adaptação às diferentes épocas. Portanto, ainda não é possível a definição da melhor época de semeadura para a região, sendo necessário pelo menos mais um ano de avaliação. A cultivar BRS 421 OL apresentou a maior massa de 100 grãos em todas as épocas de semeadura em que ela está presente, em comparação as demais cultivares. A semeadura na primeira época (07 de novembro), promoveu a maior massa de 100 grãos nas cultivares BRS 423 OL e BRS 425 OL. A cultivar EC 98 AO obteve as maiores massas quando semeada em 07 de novembro e 30 de 
janeiro. A cultivar Granoleico não obteve diferença significativa quanto a massa de 100 grãos nas diferentes épocas de semeadura.

Palavras-chave: Arachis hypogaea L.; Épocas; Produtividade; Amendoim no Mato Grosso.

\title{
Agronomic performance of peanut cultivars at different sowing times in the conditions of Sorriso-MT
}

\begin{abstract}
Growers from Mato Grosso have shown interest in the cultivation of peanuts, but the information generated by the research is still insufficient about seasons and cultivars. The objective of this study is to determine the best sowing time for 5 peanut cultivars under the conditions of Sorriso-MT. The experiment was conducted in an experimental area of the Federal Institute of Education, Science and Technology - IFMT Campus Sorriso, located in the city of Sorriso-MT. The experimental design used was in randomized blocks, each treatment with 3 repetitions, in a $5 \times 5$ factorial scheme, with 5 sowing times (11/07/2019, 11/28/2019, 12/19/2019, 9/1 / 2020 and 01/30/2020) and 5 peanut cultivars (BRS 421 OL, BRS 423 OL, BRS 425 OL, developed by Embrapa's Peanut Breeding Program and the cultivars EC 98 AO and Granoleico developed by Criadero El Carmen from Argentina The plots were composed by 4 lines of 4 meters in length and spacing of $0.90 \mathrm{~m}$ At 135 days after emergence, plants from the useful area were collected to determine the peanut yield in pods and the mass of 100 grains. experimental conditions in which this research was developed, it can be concluded that the cultivar BRS 425 $O L$ and Granoleico had the highest yields at the time of november 7, while EC 98 AO was more productive on January 30 compared to the other seasons. The cultivars BRS 421 OL and BRS 423 OL did not obtain significant difference in productivity in different sowing times, presenting similar results in all sowing times, demonstrating possible adaptation to different seasons. Therefore, it is not yet possible to define the best sowing time for the region, requiring at least another year of evaluation. The cultivar BRS 421 OL presented the largest mass of 100 grains in all the sowing seasons in which it is present, in comparison to the other cultivars. Sowing in the first season (november 7), promoted the largest mass of 100 grains in cultivars BRS 423 OL and BRS 425 OL. The cultivar EC 98 AO obtained the highest masses when sown on November 7 th and January 30th. The cultivar Granoleico showed no significant difference in terms of the mass of 100 grains at different sowing times.
\end{abstract}

Keywords: Arachis hypogaea L.; Seasons; Productivity; Peanuts in Mato Grosso.

\section{Rendimiento agronómico de los cultivares de maní en diferentes tiempos de siembra en las condiciones de Sorriso- MT}

\author{
RESUMEN
}

Los productores de Mato Grosso han mostrado interés en el cultivo de maní, pero la información generada por la investigación aún es insuficiente sobre temporadas y cultivares. El objetivo de este estudio es determinar el mejor tiempo de siembra para 5 cultivares de maní en las condiciones de Sorriso-MT. El experimento se realizó en un área experimental del Instituto Federal de Educación, Ciencia y Tecnología - IFMT Campus Sorriso, ubicado en la ciudad de Sorriso-MT. El diseño experimental utilizado fue en bloques al azar, cada tratamiento con 3 repeticiones, en un esquema factorial 5 x 5, con 5 tiempos de siembra (07/11/2019, 28/11/2019, 19/12/2019, 1/09 / 2020 y 30/01/2020) y 5 cultivares de maní (BRS 421 OL, BRS 423 OL, BRS 425 OL, desarrollados por el Programa de Mejoramiento de Maní de Embrapa y los cultivares EC 98 AO y Granoleico desarrollados por Criadero El Carmen de Argentina Las parcelas estaban compuestas por 4 líneas de 4 metros de largo y espaciamiento de $0.90 \mathrm{~m}$. A los 135 días después de la emergencia, se cosecharon plantas del área útil para determinar el rendimiento de maní en vaina y la masa de 100 granos. En las condiciones experimentales en las que se desarrolló esta investigación, se puede concluir que el cultivar BRS 425 OL y Granoleico tuvieron los mayores rendimientos al momento del 7 de noviembre, mientras que EC 98 AO fue más productivo el 30 de enero en comparación con otras temporadas. Los cultivares BRS 421 
OL y BRS 423 OL no obtuvo diferencia significativa en la productividad en diferentes tiempos de siembra, presentando resultados similares en todos los tiempos de siembra, demostrando posible adaptación a diferentes épocas. Por lo tanto, aún no es posible definir el mejor momento de siembra para la región, requiriendo al menos otro año de evaluación. El cultivar BRS 421 OL presentó la mayor masa de 100 granos en todas las temporadas de siembra en las que está presente, en comparación con los demás cultivares. La siembra en la primera temporada (7 de noviembre) promovió la mayor masa de 100 granos en los cultivares BRS 423 OL y BRS 425 OL. El cultivar EC 98 AO obtuvo las mayores masas cuando se sembró el 7 de noviembre y el 30 de enero. El cultivar Granoleico no mostró diferencias significativas en cuanto a la masa de 100 granos en diferentes tiempos de siembra.

Palabras clave: Arachis hypogaea L.; Estaciones; Productividad; Maní en Mato Grosso.

\section{Introdução}

De acordo com o Departamento de Agricultura dos Estados Unidos (2020), a produção brasileira de amendoim (Arachis hypogaea L.) é considerada apenas $1,2 \%$ da produção mundial. O estado de São Paulo é o maior produtor nacional, sendo responsável por 99,3\% das exportações brasileiras de amendoim. Os principais destinos desta produção são os países da Rússia (33\%), Argélia (18\%), Holanda (11\%), Ucrânia (5,5\%), Polônia (4,2\%) e Colômbia (3,9\%), (MDIC, 2020). No cenário das exportações, o Brasil se destaca como quinto maior exportador mundial, (MASSAFERA, 2020) com um volume de 125 mil toneladas e crescimento de $27,6 \%$ em comparação ao mesmo período do ano passado (98 mil toneladas) (MDIC, 2020).

No estado de São Paulo, a cultura do amendoim tem enfrentado condições edafoclimáticas as vezes desfavoráveis, elevado custo do arrendamento das terras. Utilizam-se cultivares de ciclo médio a tardio (130 a 140 dias) em áreas de renovação de canaviais que ficam disponíveis por 130 dias, logo há competição pela área com a soja que leva vantagem, pois tem um ciclo mais rápido e se encaixa melhor neste intervalo. Neste sentido, a expansão da cultura do amendoim para outras regiões do país, é primordial para o Brasil alcançar maior representatividade no mercado internacional (SUASSUNA et al., 2018). É necessário testar o desempenho de cultivares de amendoim selecionadas em diferentes regiões do Brasil, de modo a obter resultados, visando atender a demanda do mercado de sementes comerciais (UITDEWILLIGEN et al., 2017) e explorar regiões 
promissoras ao cultivo do amendoim, como no estado de Mato Grosso (RIZZI et al., 2019; SANTIN et al., 2019).

Dessa forma, a região Centro Oeste, especialmente o médio norte de Mato Grosso, apresenta reconhecida aptidão agrícola, terras favoráveis ao cultivo e a mecanização em larga escala, clima bem definido, produtores ávidos por alternativas econômicas voltadas ao mercado externo, o que pode contribuir para a expansão do cultivo de amendoim no Brasil (LAZAROTTO, 2020).

Produtores do Mato Grosso tem demonstrado interesse no cultivo do amendoim, pois tem-se áreas que permitem a expansão da cultura, fato citado por Migliari (2020) não ocorre na Argentina, que é referência no cultivo de amendoim. Essa situação coloca a região Médio Norte em um cenário animador ao estabelecimento e fortalecimento de toda a cadeia produtiva do amendoim, com a possível instalação de uma unidade de beneficiamento na região, para dar o devido suporte e processamento a produção local.

Para tanto, é preciso conhecer o potencial produtivo do amendoim na região e o quanto deste potencial vai ser expresso mediante os fatores limitantes que atuam durante o ciclo da cultura, em diferentes condições de temperatura e umidade. A definição da época de semeadura depende de um conjunto de fatores ambientais que, além de afetar a produtividade, afeta também a arquitetura e o desenvolvimento da planta. Semeaduras em épocas inadequadas podem causar reduções drásticas na produtividade (PEIXOTO et al., 2002). Nesse sentido, o objetivo deste estudo é determinar a melhor época de semeadura para 5 cultivares de amendoim nas condições de Sorriso-MT.

\section{Material e Métodos}

O experimento foi conduzido em área experimental do Instituto Federal de Educação, Ciência e Tecnologia - IFMT Campus Sorriso, localizada à latitude $12^{\circ} 41^{\prime} 43^{\prime \prime} \mathrm{S}$, longitude $55^{\circ} 48^{\prime} 07^{\prime \prime}$ W e altitude média de 
$358 \mathrm{~m}$, em solo classificado como Latossolo Vermelho Amarelo distrófico com textura argilosa. O clima da região é tipo Aw, com temperatura média de $26,2^{\circ} \mathrm{C}$ e pluviosidade média de $1970 \mathrm{~mm}$ anuais os quais são distribuídos nos meses de outubro a abril (SOUZA et al., 2013).

O delineamento experimental utilizado foi em blocos casualizados, cada tratamento com 3 repetições, em esquema fatorial $5 \times 5$, sendo 5 épocas de semeadura (07/11/2019, 28/11/2019, 19/12/2019, 09/01/2020 e 30/01/2020) e 5 cultivares de amendoim (BRS 421 OL, BRS 423 OL, BRS $425 \mathrm{OL}$, desenvolvidas pelo Programa de Melhoramento do Amendoim da Embrapa e as cultivares EC 98 AO e Granoleico desenvolvidas pelo Criadero El Carmen da Argentina. As parcelas foram compostas por 4 linhas de 4 metros de comprimento e espaçamento de $0,90 \mathrm{~m}$. As duas linhas centrais foram consideras como área útil, totalizando $7,2 \mathrm{~m}^{2}$ por parcela. $\mathrm{A}$ cultivar BRS 421 OL, não foi utilizada na primeira e na última época de semeadura devido a não disponibilidade de sementes.

Antecedendo a implantação do experimento, foi efetuado o levantamento da fertilidade e granulometria do solo, utilizando-se metodologia proposta por Raij et al. (2001) e Embrapa (1997), na profundidade de 0 a $0,20 \mathrm{~m}$. Os resultados das análises foram: $\mathrm{pH}\left(\mathrm{CaCl}_{2}\right)$ 5,30; P (resina) 5,0 mg/dm $\mathrm{dm}^{3}$ K $78 \mathrm{mg} / \mathrm{dm}^{3}$; Zn $1,0 \mathrm{mg} / \mathrm{dm}^{3}$; Fe $58 \mathrm{mg} / \mathrm{dm}^{3}$;

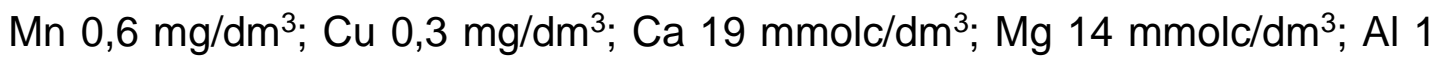
mmolc/ $\mathrm{dm}^{3}$ e $\mathrm{H}+\mathrm{Al} 40 \mathrm{mmolc} / \mathrm{dm}^{3}$ com saturação por bases $46 \%$ e matéria orgânica 2,0\%. A análise granulométrica de areia, silte e argila apresentou 434, 26 e $540 \mathrm{~g} \mathrm{dm}^{-3}$, respectivamente. Visando aumentar a saturação por bases para $60 \%$, foi realizado calagem de 1,4 ton ha ${ }^{-1}$ de calcário dolomítico, 60 dias antes da semeadura da primeira época, sendo este incorporado com 2 gradagens.

Em todas as épocas, as sementes foram tratadas com carboxina + tiram e distribuídas na densidade de 28 sementes por metro (germinação $90 \%$ ). A adubação de base consistiu na aplicação de $300 \mathrm{~kg} \mathrm{ha}^{-1}$ do formulado NPK 04-30-10, distribuído no sulco de semeadura. Em cobertura, aos 35 dias após a emergência (DAE), foi realizada a aplicação de $500 \mathrm{~kg}$ 
ha $^{-1}$ de gesso agrícola, aplicado a lanço em área total. $O$ manejo fitossanitário foi feito conforme a recomendação para a cultura.

Aos 135 DAE foram colhidas as plantas da área útil para determinação da produtividade de amendoim em vagens $\left(\mathrm{kg} \mathrm{ha}^{-1}\right)$ (corrigido a $8 \%$ de umidade) e a massa de 100 grãos (g). Os dados foram submetidos à análise de variância pelo teste $\mathrm{F}$ e as médias dos tratamentos foram agrupados pelo teste de Scott-Knott a 1 e $5 \%$ de probabilidade utilizando o programa estatístico SISVAR (FERREIRA, 2019).

\section{Resultados e discussão}

Após a análise de variância foram observados efeitos significativos para épocas de semeadura, cultivares e interação entre os fatores para as variáveis produtividade de vagens $\left(\mathrm{kg} \mathrm{ha}^{-1}\right)$ e massa de 100 grãos $(\mathrm{g}$ ) conforme a Tabela 1. Desta forma os dados foram desdobrados para massa de 100 grãos (g) (Tabela 2) e produtividade de vagens $\left(\mathrm{kg} \mathrm{ha}^{-1}\right)$ (Tabela 3).

Tabela 1. Resumo da análise de variância para massa de 100 grãos (g) (M100) e produtividade de vagens ( $\mathrm{kg} \mathrm{ha}^{-1}$ ) (PROD) em função de cultivares de amendoim em diferentes épocas de semeadura. Sorriso-MT, 2019/20.

\begin{tabular}{lcc}
\hline \multirow{2}{*}{ Fontes de variação } & \multicolumn{2}{c}{ Quadrados Médios } \\
\cline { 2 - 3 } & $\mathrm{M} 100(\mathrm{~g})$ & $\mathrm{PROD}\left(\mathrm{kg} \mathrm{ha}^{-1}\right)$ \\
\hline Cultivares $(\mathrm{A})$ & $341,5^{\star *}$ & $1.638 .460,9^{\star *}$ \\
Épocas de semeadura (B) & $292,8^{*}$ & $1.793 .128,6^{* *}$ \\
Interação $(\mathrm{A} \times \mathrm{B})$ & $104,7^{\star *}$ & $439.738,9^{\star *}$ \\
Coeficiente de variação (\%) (A) & 7,72 & 9,04 \\
Coeficiente de variação (\%) (B) & 11,52 & 6,21 \\
\hline ns não significativo; * e ${ }^{* *}$ significativo a 5 e $1 \%$ de probabilidade, \\
respectivamente.
\end{tabular}

$\mathrm{Na}$ Tabela 2 estão apresentados os resultados do desdobramento para a variável produtividade de vagens $\left(\mathrm{kg} \mathrm{ha}^{-1}\right)$. Observa-se que na semeadura realizada em 07 de novembro, a produtividade de vagens não foi estatisticamente diferente entre as cultivares BRS $425 \mathrm{OL}\left(5.747,1 \mathrm{~kg} \mathrm{ha}^{-1}\right)$, BRS $423 \mathrm{OL}\left(5.554,9 \mathrm{~kg} \mathrm{ha}^{-1}\right)$ e Granoleico $\left(5.454,7 \mathrm{~kg} \mathrm{ha}^{-1}\right)$, porém estas foram significativamente superiores a cultivar EC $98 \mathrm{AO}\left(4.103,8 \mathrm{~kg} \mathrm{ha}^{-1}\right)$. 
Tabela 2. Produtividade de vagens $\left(\mathrm{kg} \mathrm{ha}^{-1}\right)$ em função de cultivares de amendoim em diferentes épocas de semeadura. Sorriso-MT, 2019/20.

\begin{tabular}{cccccc}
\hline \multirow{2}{*}{ Cultivares } & \multicolumn{5}{c}{ Épocas de Semeadura } \\
\cline { 2 - 6 } & $\mathbf{0 7}$ novembro & $\mathbf{2 8}$ novembro & $\mathbf{1 9}$ dezembro & $\mathbf{0 9}$ janeiro & $\mathbf{3 0}$ janeiro \\
\hline BRS 421 OL & - & $4.471,0 \mathrm{aA}$ & $4.743,3 \mathrm{aA}$ & $4.382,5 \mathrm{aA}$ & - \\
BRS 423 OL & $5.554,9 \mathrm{aA}$ & $5.123,8 \mathrm{aA}$ & $4.671,2 \mathrm{aA}$ & $4.971,3 \mathrm{aA}$ & $5.042,5 \mathrm{aA}$ \\
BRS 425 OL & $5.747,1 \mathrm{aA}$ & $4.731,4 \mathrm{aB}$ & $4.600,9 \mathrm{aB}$ & $4.332,1 \mathrm{aB}$ & $5.020,8 \mathrm{aB}$ \\
EC 98 AO & $4.103,8 \mathrm{bB}$ & $3.671,4 \mathrm{bB}$ & $4.333,7 \mathrm{aB}$ & $4.127,9 \mathrm{aB}$ & $4.845,3 \mathrm{aA}$ \\
Granoleico & $5.454,7 \mathrm{aA}$ & $3.483,5 \mathrm{bC}$ & $4.778,8 \mathrm{aB}$ & $4.384,2 \mathrm{aB}$ & $4.728,1 \mathrm{aB}$ \\
\hline
\end{tabular}

Letras iguais minúsculas na coluna e maiúsculas na linha pertencem ao mesmo grupo, de acordo com o teste Scott-Knott à 5\% de probabilidade.

Para a semeadura em 28 de novembro, as cultivares BRS 423 OL (5.123,8 kg ha $\left.{ }^{-1}\right)$, BRS 425 OL $\left(4.731,4 \mathrm{~kg} \mathrm{ha}^{-1}\right)$ e BRS $421 \mathrm{OL}(4.471,0 \mathrm{~kg}$ $\left.\mathrm{ha}^{-1}\right)$ não apresentaram diferença de produtividade entre si, mas foram estatisticamente superiores as cultivares EC 98 AO $\left(3.671,4 \mathrm{~kg} \mathrm{ha}^{-1}\right) \mathrm{e}$ Granoleico $\left(3.483,5 \mathrm{~kg} \mathrm{ha}{ }^{-1}\right)$. Não houve diferença significativa para produtividade de vagens entre as cultivares para as épocas de semeadura 19 de dezembro, 09 de janeiro e 30 de janeiro. Somente as cultivares BRS 421 OL e BRS 423 OL não apresentaram diferença significativa na produtividade entre as épocas de semeadura.

Nesse sentido, observa-se que a cultivar EC 98 AO demostrou maior produtividade para a semeadura mais tardia, em 30 de janeiro $(4.845,3 \mathrm{~kg}$ $\mathrm{ha}^{-1}$ ), sendo significativamente maior em relação as produtividades obtidas nas épocas anteriores. E a cultivar Granoleico obteve maior produtividade quando foi semeada em 07 de novembro $\left(5.454,7 \mathrm{~kg} \mathrm{ha}^{-1}\right)$, em comparação as demais épocas. De forma generalizada, constata-se que a época de semeadura de 07 de novembro foi a que permitiu maiores produtividades de forma significativa para as cultivares BRS 425 OL e Granoleico.

Quanto a época de semeadura 09 de janeiro, corrobora-se com Rizzi et al. (2019), que testando o desempenho agronômico de diferentes cultivares de amendoim (BRS 421 OL, BRS 423 OL, BRS 425 OL, EC 98 AO e IAC OL 3), não obtiveram diferença significativa na produtividade de vagens, com semeadura realizada 09 de janeiro de 2019, em Sorriso-MT. Na 
época de semeadura 07 de novembro, discorda-se de Santin et al. (2019), testando o desempenho agronômico de diferentes cultivares de amendoim (BRS 421 OL, BRS 423 OL, BRS 425 OL, IAC 503 e IAC OL 3), não obtiveram diferença significativa na produtividade de vagens, com semeadura realizada 05 de novembro de 2019, em Campo Verde-MT.

Contudo, ressalta-se que em semeaduras muito tardias, o processo de arranquio do amendoim coincide com o período de baixa umidade do solo, o que dificulta e aumenta os custos do arranquio, além de proporcionar maiores perdas de vagens no solo. A semeadura entre 28 de novembro e 09 de janeiro, em observações visuais, percebeu-se a incidência maior de doenças, em especial as causadas pelos fungos Cercosporidium personatum e Sclerotium rolfsii nos cultivares EC 98 AO e Granoleico, o que pode ter contribuído para as menores produtividade de vagens.

O desempenho da cultura em semeadura tardia (30 de janeiro), demonstrou resultados interessantes, podendo abrir a possibilidade do cultivo em safrinha, após o cultivo da soja. Para esta época de semeadura, foi observado a redução de 20 dias no ciclo total, obtendo-se produtividade similar ou até maior às obtidas nas épocas anteriores. Contudo, vale ressaltar que a precipitação pluviométrica no período foi atípica, sendo 100 $\mathrm{mm}$ abaixo da média em fevereiro e $150 \mathrm{~mm}$ acima da média em março, o que pode ter contribuído para a maior disponibilidade de água no período de floração e enchimento de grãos, proporcionando bons níveis de produtividade.

$\mathrm{Na}$ Tabela 3 estão apresentados os resultados do desdobramento para a variável massa de 100 grãos (g). Observa-se que na primeira época de semeadura (07 de novembro), as cultivares BRS $425 \mathrm{OL}$ (79,9 g) e BRS $423 \mathrm{OL}(76,6 \mathrm{~g})$ obtiveram as maiores massas de 100 grãos em comparação as cultivares EC 98 AO $(70,5 \mathrm{~g})$ e Granoleico $(69,4 \mathrm{~g})$. Nas três épocas de semeadura seguintes (28 de novembro, 19 de dezembro e 09 de janeiro) a cultivar BRS $421 \mathrm{OL}$ apresentou à maior massa de 100 grãos, sendo superior à média dos outros cultivares em todas as épocas, com as respectivas massas: 77,4; 75,1 e 75,0 g. A maior massa de grãos obtida no 
cultivar BRS 421 OL também foi observada por Ribeiro et al. (2017) e Domenici et al. (2018) em experimentos conduzidos em Tupã-SP e Frutal$M G$, respectivamente. Para a semeadura tardia, em 30 de janeiro, não houve diferença na massa de 100 grãos entre as cultivares.

Tabela 3. Massa de 100 grãos (g) em função de cultivares de amendoim em diferentes épocas de semeadura. Sorriso-MT, 2019/20.

\begin{tabular}{cccccc}
\hline \multirow{2}{*}{ Cultivares } & \multicolumn{5}{c}{ Épocas de semeadura } \\
\cline { 2 - 6 } & 07 novembro & 28 novembro & 19 dezembro & 09 janeiro & 30 janeiro \\
\hline BRS 421 OL & - & $77,4 \mathrm{aA}$ & $75,1 \mathrm{aA}$ & $75,0 \mathrm{aA}$ & - \\
BRS 423 OL & $76,6 \mathrm{aA}$ & $68,2 \mathrm{bB}$ & $61,2 \mathrm{bB}$ & $62,4 \mathrm{bB}$ & $63,2 \mathrm{aB}$ \\
BRS 425 OL & $79,9 \mathrm{aA}$ & $69,5 \mathrm{bB}$ & $54,5 \mathrm{bC}$ & $59,0 \mathrm{bC}$ & $59,4 \mathrm{aC}$ \\
EC 98 AO & $70,5 \mathrm{bA}$ & $50,7 \mathrm{~dB}$ & $60,9 \mathrm{bB}$ & $57,1 \mathrm{bB}$ & $65,8 \mathrm{aA}$ \\
Granoleico & $69,4 \mathrm{bA}$ & $59,3 \mathrm{cA}$ & $64,3 \mathrm{bA}$ & $62,1 \mathrm{bA}$ & $61,2 \mathrm{aA}$ \\
\hline
\end{tabular}

Letras iguais minúsculas na coluna e maiúsculas na linha pertencem ao mesmo grupo, de acordo com o teste Scott-Knott à 5\% de probabilidade.

As cultivares BRS 421 OL e Granoleico não apresentaram diferença significativa na massa de 100 grãos entre as épocas de semeadura. A cultivar EC $98 \mathrm{AO}$ demostrou maior massa de grãos quando semeada em 07 de novembro ( $70,5 \mathrm{~g})$ e 30 de janeiro $(65,8 \mathrm{~g})$, sendo significativamente maiores em relação as massas obtidas nas demais épocas.

\section{Conclusões}

Nas condições experimentais em que foi desenvolvida esta pesquisa, pode-se concluir que a cultivar BRS $425 \mathrm{OL}$ e Granoleico obtiveram as maiores produtividades na época de 07 de novembro, enquanto a EC 98 AO foi mais produtiva em 30 de janeiro em comparação às demais épocas de semeadura. As cultivares BRS $421 \mathrm{OL}$ e BRS $423 \mathrm{OL}$ não obtiveram diferença significativa quanto a produtividade nas diferentes épocas de semeadura, apresentando resultados similares em todas as épocas de semeadura, demonstrando possível adaptação às diferentes épocas. Portanto, ainda não é possível a definição da melhor época de semeadura para a região, sendo necessário pelo menos mais um ano de avaliação. A cultivar BRS $421 \mathrm{OL}$ apresentou a maior massa de 100 grãos 
em todas as épocas de semeadura em que ela está presente, em comparação as demais cultivares. A semeadura na primeira época (07 de novembro), promoveu a maior massa de 100 grãos nas cultivares BRS 423 OL e BRS 425 OL. A cultivar EC 98 AO obteve as maiores massas quando semeada em 07 de novembro e 30 de janeiro. A cultivar Granoleico não obteve diferença significativa quanto a massa de 100 grãos nas diferentes épocas de semeadura.

\section{Agradecimentos}

Os autores agradecem aos demais discentes do curso de Engenharia Agronômica que contribuíram na condução do experimento. A parceria institucional com o Programa de Melhoramento do Amendoim Embrapa, vinculado ao projeto SEG 20.18.01.021.00, LC Sementes, MIAC Indústrias Colombo e a Beatrice Peanuts. 


\section{Referências}

Departamento da Agricultura dos Estados Unidos. World Agricultural Producion. Foreign Agricultural Service, wap 5-20, p. 32, jul. 2020.

DOMENICI, M. G.; ZACHARIAS, A. O.; GUIRALDELLI, E. H.; HEUERT, J.; SUASSUNA, T. M. F. Desempenho de genótipos de amendoim no Triângulo Mineiro. In: Anais do encontro sobre a cultura do amendoim, 15., 2018, Jaboticabal. Anais eletrônicos... Campinas, GALOÁ, 2018. Disponível em: $<$ https://proceedings.science/encontro-amendoim-2018/papers/desempenhodegenotipos-de-amendoim-no-triangulo-mineiro>. Acesso em: 20 jul. 2020.

EMBRAPA. Centro Nacional de Pesquisa de Solos (Rio de Janeiro, RJ). Manual de métodos de análise de solo. 2.ed. Rio de Janeiro EmbrapaCNPS, 1997.212p.

FERREIRA, D. F. SISVAR: um sistema de análise de computador para efeitos fixos projetos de tipo de partida dividida. Revista Brasileira de Biometria, v. 37, n. 4, p. 529-535, 2019.

LAZAROTTO, C. Referência no cultivo e beneficiamento do amendoim, empresários paulistas estão de olho em Sorriso. 2020. Disponível em: $<$ https://site.sorriso.mt.gov.br/noticia/referencia-no-cultivo-e-beneficiamentodo-amendoim-empresarios-paulistas-estao-de-olho-em-sorriso5e6248f3d321d>. Acesso em: 25 jul 2020.

MASSAFERA, R. Amendoim brasileiro pode ter safra recorde este ano. 2020. Disponível em: <https://www.biodieselbr.com/noticias/materiaprima/outras/amendoim-brasileiro-pode-ter-safra-recorde-este-ano-160320>. Acesso em: 25 jul. 2020.

MDIC. Amendoins. 2020. Disponível em: $<$ http://comexstat.mdic.gov.br/pt/comex-vis>. Acesso em: 25 jul 2020.

MIGLIARI, A. C. Argentinos conhecem Mato Grosso para investir em amendoim. [entrevista concedida a] Redação. O documento, Mato Grosso, 2020. Disponível em <https://odocumento.com.br/argentinos-conhecemmato-grosso-para-investir-em-amendoim/>. Acesso em: 13 maio 2020.

PEIXOTO, C. P.; CAMARA G. M. S.; MARTINS, M. C.; MARCHIORI, L. F. S. Efeitos de épocas de semeadura e densidade de plantas sobre a produtividade de cultivares de soja no Estado de São Paulo. Revista de Agricultura, Piracicaba, v. 77, n. 2, p. 265-291, 2002.

RAIJ, B.; ANDRADE, J. C.; CANTARELLA, H.; QUAGGIO, J. A. Análise química para avaliação da fertilidade de solos tropicais. Campinas Instituto Agronômico, 2001. 285p. 
RIBEIRO, R. P.; HEUERT, L.; SUASSUNA, N. D.; SUASSUNA, T. M. F. Desempenho de linhagens de amendoim sob alta severidade de doenças foliares. In: Anais do encontro sobre a cultura do amendoim, 14., 2017, Jaboticabal. Anais eletrônicos... Campinas, GALOÁ, 2018. Disponível em: $<$ https://proceedings.science/encontro-amendoim-2017/papers/desempenhode-linhagens-de-amendoim-sob-alta-severidade-de-doencas-foliares>. Acesso em: 18 abr. 2020.

RIZZI, T. S.; OLIBONE, D.; LODEA, L.; HEUERT, J.; XAVIER, M. F. N.; SUASSUNA, T. M. F. Desempenho de cultivares de amendoim na região Médio-Norte Mato-Grossense. In: Anais do encontro sobre a cultura do amendoim, 16., 2019, Jaboticabal. Anais eletrônicos... Campinas, GALOÁ, 2019. Disponível em: <https://proceedings.science/encontro-amendoim2019/papers/desempenho-de-cultivares-de-amendoim-na-regiao-medionorte-mato-grossense>. Acesso em: 25 jul. 2020.

SANTIN, V.; PEROZINI, A. C.; ARAÚJO, C.; GIRON, F. G.; HEUERT, J.; XAVIER, M. F. N.; SUASSUNA, T. M. F. Desempenho de cultivares de amendoim nas condições de Campo Verde-MT. In: Anais do encontro sobre a cultura do amendoim, 16., 2019, Jaboticabal. Anais eletrônicos... Campinas, GALOÁ, 2019. Disponível em: $<$ https://proceedings.science/encontro-amendoim-2019/papers/desempenhode-cultivares-de-amendoim-nas-condicoes-de-campo-verde-mt>. Acesso em: 25 jul. 2020.

SOUZA, A. P.; MOTA, L. L.; ZAMADEI, T. MARTIM, C. C.; ALMEIDA, F. T.; PAULINO, J. Classificação climática e balanço hídrico climatológico no estado de Mato Grosso. Nativa, v. 01, n. 01, p. 34-43, 2013.

SUASSUNA, T. M. F.; HEUERT, J.; BOGGIANI, J. C.; PERINA, F. J.; SOFIATTI, V.; BETTINI, P. C.; OLIVEIRA, M. C. T.; LEONEL, C. L. Desempenho de linhagens de amendoim na região do Cerrado. In: Anais do encontro sobre a cultura do amendoim, 15., 2018, Jaboticabal. Anais eletrônicos... Campinas, GALOÁ, 2018. Disponível em: $<$ https://proceedings.science/encontro-amendoim-2018/papers/desempenhode-linhagens-de-amendoim-na-regiao-do-cerrado>. Acesso em: 25 jul. 2020.

UITDEWILLIGEN, G. S.; REICH, T. C.; CAPORUSSO, N. B.; SUASSUNA, N. D.; HEUERT, J.; SUASSUNA, T. M. F. Avaliação do desempenho de linhagens de amendoim em Jaboticabal, São Paulo, via modelos mistos. In: Anais do encontro sobre a cultura do amendoim, 14., 2017, Jaboticabal. Anais eletrônicos... Campinas, GALOÁ, 2017. Disponível em: <https://proceedings.science/encontro-amendoim-2017/papers/avaliacao-dodesempenho-de-linhagens-de-amendoim-em-jaboticabal--sao-paulo--viamodelos-mistos>. Acesso em: 25 jul. 2020. 